\title{
Blockade of Osteopontin Inhibits Glomerular Fibrosis in a Model of Anti-Glomerular Basement Membrane Glomerulonephritis
}

\author{
Cindy Zhou ${ }^{\mathrm{a}}$ Jean Wu $\mathrm{u}^{\mathrm{a}}$ Lisa Torres $^{\mathrm{a}} \quad$ John M. Hicks ${ }^{\mathrm{b}}$ Todd Bartkowiak $^{\mathrm{a}}$ \\ Kiana Parker ${ }^{a}$ Ya-Huan Lou ${ }^{a}$ \\ a Department of Diagnostic Sciences DB, University of Texas Health Science Center at Houston, and \\ ${ }^{\text {b}}$ Texas Children's Hospital, Houston, Tex., USA
}

Key Words

Fibrosis · Extracellular matrix · Glomerulonephritis ·

Animal models

\begin{abstract}
Background: In our rat model for anti-GBM GN, severe fibrosis follows glomerular inflammation. A potential role of extracellular matrix protein osteopontin (OPN) in glomerular fibrosis was investigated. Methods: Neutralizing OPN antiserum or control normal serum was injected into the experimental rats at late inflammatory/early fibrotic stage. Glomerular inflammation and fibrosis were determined. Results: OPN antiserum treatment had little effect on glomerular inflammation. However, the antiserum treatment resulted in a significant reduction in number of fibrotic glomeruli $50 \%$ of the controls). Histology observation showed that fibrotic tissue in glomeruli of the antiserum treated rats was mild and poorly developed. OPN antiserum treatment resulted in downregulated glomerular expression of collagen $1 \alpha 1$; collagen deposition in the antiserum treated rats reduced to $<30 \%$ of that for normal serum controls. Conclusion: Neutralization of OPN inhibited progression of fibrosis in vivo when given at early fibrotic stage. Thus, OPN may be a therapeutic target for glomerular fibrosis.
\end{abstract}

Copyright $\odot 2010$ S. Karger AG, Basel
๑) 2010 S. Karger AG, Basel

Fax +41613061234

E-Mail karger@karger.ch

www.karger.com
Accessible online at: www.karger.com/ajn

\section{Introduction}

End-stage renal disease is characterized by the development of fibrotic lesions in the glomerular and other renal compartments. Many diseases such as diabetes, hypertension and various inflammatory glomerulonephritis (GN) eventually lead to glomerular fibrosis, which permanently destroys glomerular architecture. Intensive studies in the past decades have shown glomerular fibrosis to be a very complicated process, involving many molecules and cells [1-4]. Similar to fibrosis occurring in other organs, TGF- $\beta /$ Smad pathway plays a central role in mesangial cell activation and differentiation of fibroblasts, which in turn produce extracellular matrix (ECM) proteins [5-7]. It is less clear how the ECM proteins, once produced, are incorporated into the architecture of fibrotic tissue and whether those proteins can regulate gene expression in fibroblasts.

Osteopontin (OPN), or SPP1, is a highly modified integrin-binding ECM glycophosphoprotein. OPN participates in many physiological and pathological events, including maintenance or re-establishment of tissue integrity, inflammatory processes and tumor metastasis [7-9]. OPN also functions as both an anti- and pro-inflammatory molecule in autoimmune pathogenesis, as well as in other immune responses [10]. More recently, a critical

Dr. Ya-Huan Lou

Department of Diagnostic Science, Dental Branch

University of Texas Houston Health Science Center

Houston, TX 77030 (USA)

Tel. +1 713500 4059, Fax +1 713500 4500, E-Mail Yahuan.Lou@uth.tmc.EDU 
role of OPN in fibrosis has been reported [11-13]. One study showed that OPN is required for differentiation of myofibroblasts, a major player in fibrosis [14]. In addition to its regulatory function, OPN is an important structural molecule.

The role of OPN in both renal development and disease has been explored $[15,16]$. Constitutive expression of $\mathrm{OPN}$ in renal tubular epithelium is required for prevention of kidney stone formation. Roles of OPN in renal fibrosis, especially in renal interstitial tissue, have been well investigated in OPN null mice. OPN may modulate angiotensin II-induced inflammation, oxidative stress, and fibrosis of the kidney [17]. Several studies reported an association of upregulation of glomerular OPN expression with macrophage accumulation and progressive renal injury $[18,19]$. Anti-OPN antibody treatment reduced proteinuria and prevented loss of renal function, suggesting OPN to be a pro-inflammatory molecule [20]. However, experiments using OPN null mice showed a lack of any in vivo functions of OPN in an anti-GBM GN model [21]. Although it still lacks direct evidence, several studies have suggested a potential role of OPN in glomerular fibrosis $[22,23]$.

We have established a rat model for autoimmune antiGBM GN, which is induced by immunization with a T cell epitope $\mathrm{pCol}(28-40)$ derived from $\alpha 3$ chain of type IV collagen $(\mathrm{Col} 4 \alpha 3)[24,25]$. The animals undergo two distinct overlapping stages after disease induction: (1) CD4+ $\mathrm{T}$ cell-mediated glomerular inflammation followed by (2) severe glomerular fibrosis. We have previously reported upregulation of OPN in a novel multinucleated myofibrobalsts in the glomeruli and massive deposition of OPN on the fibrotic tissue during fibrotic stage [26]. In the present study, we investigated whether blockage of OPN activity at late inflammatory/early fibrotic stage would alter fibrosis progress in our model.

\section{Methods}

\section{Antigen Preparation and GN Induction}

Nephritogenic T cell peptide pCol(28-40) was commercially synthesized and dissolved in milli-Q water at a 1-mM concentration. Female Wistar Kyoto (WKY) rats (4-6 weeks of age) were purchased from Harlan (Indianapolis, Ind., USA). The rats were maintained in the animal facility at the University of Texas Houston Health Science Center and allowed to acclimate for a minimum of 3 days. Rats were immunized with a peptide $(0.125 \mu \mathrm{mol})$ emulsified in CFA, in one hind footpad and at the base of the tail. Rats immunized with CFA alone served as controls. All animal procedures were approved by the IACUC.

Osteopontin Blockage Inhibits

Glomerular Fibrosis
Evaluation of GN Severity and Glomerular Fibrosis

Urine samples were collected daily for evaluation of albuminuria. In some cases, 24-hour urine was collected using metabolism cages and urine albumin concentrations were determined with a Rat Albumin ELISA Quantitation Set (Bethyl Lab, Montgomery, Tex., USA). GN severity in experimental rats was graded by histology. A portion of kidney tissues were fixed in Bouin's solution, and glomerular injury score was calculated as described previously [24]. The tissue sections were processed for PAS, trichrome staining. Glomeruli with fibrotic crescent lesions were identified and expressed as a percentage of all glomeruli. Tissue sections stained with anti-collagen $1 \alpha$ lantibody were used for calculation of collagen positive area in glomeruli with a program (Nikon). A portion of renal tissue was processed for transmission electron microscopy [24]. Glomerular fibrosis was also evaluated by RT-PCR on expression of collagen $1 \alpha 1$ following a previously published method [5]. In addition to acidic ribosomal phosphoprotein P0, housekeeper gene GAPDH was also used as another control. Real-time PCR was performed for the collagen $1 \alpha 1$ gene using $\mathrm{P} 0$ as a comparison.

In vivo Blockage of OPN Activity with OPN Antibody

A rabbit anti-OPN polyclonal antibody, which has been demonstrated to react with OPN and block its activity, was provided by Dr. William Butler, a pioneer in OPN research, University of Texas Health Science Center at Houston. A normal rabbit serum (NRS) was used as a negative control. Rats were immunized with pCol(2840). A total of $800 \mu \mathrm{l}$ of antisera or normal sera were injected i.v. into each immunized rat at days $26,28,33$ and 38 postimmunization. The rats were sacrificed at day 40 or day 50 , or at their death. Kidneys of 3 rats from each group were used for isolation of glomerular cells at days 40 and 50 . The cells were analyzed by flow cytometry or for overnight culture to detect fibroblast-like cells in wells of a 96-well plate. Approximately 6-7 pictures were taken to cover an entire well under $\times 100$ magnification. Fibroblast spread area was determined by computer-assisted area calculation (NISElement 2.3, Nikon). A portion of the kidney was fixed for histological evaluation. Sera OPN concentrations were determined by Rat OPN Assay Kit from Immuno-Biological Laboratory (IBL, Guma, Japan). Rabbit IgG concentration in recipients' sera were measured by ELISA using a pair of polyclonal anti-rabbit IgG antibodies (Bethyl Lab, Montgomery, Tex., USA). OPN-mediated peritoneal macrophage invasion assay was performed following a published method with minor modifications. Rat recombinant MCP-1 (R\&D, Minneapolis, Minn., USA) was used as a chemoattractant.

Purification and Analysis of Glomerular Cells

Glomerular cells were isolated at days 40 or 50 postimmunization for detection of OPN expression and determination of leukocyte populations. After kidneys were perfused in situ with PBS, glomeruli were isolated to a high purity as previously described using repeated low-speed centrifugations. The isolated glomeruli with purity over $95 \%$ were digested with a mixture of collagenase IV and AccutaseTM (eBioscience, San Diego, Calif., USA) at room temperature for $30 \mathrm{~min}$ with gentle stirring. The cells were released by repeated pipetting and collected by centrifugation. Dead cells were further removed by Ficoll density centrifugation. The cells were used for immunofluorescence, flow cytometry or further culture with antibody or normal sera. For immunofluoresence, the cells were placed in each well of a 4 -well chamber slide 
overnight (Fisher, Rochester, N.Y., USA). For flow cytometry, the isolated cells were stained with paired antibodies (PE-anti-CD4 (OX4) vs. FITC-anti-rat IgG, or PE-anti-CD8 (OX8) vs. FITC-anti-CD11b/c). The stained cells were analyzed with a flow cytometer (FACSCalibur, Becton Dickinson, San Jose, Calif., USA). Glomerular fibroblasts were isolated from early stage of fibrosis. In some cases, attached fibroblasts were collected by trypsin digestion and used for other purposes. Fibroblast proliferation was determined by the Quick cell proliferation Assay Kit (BioVision).

Statistical Analysis

One-way ANOVA test was used for comparisons between experimental groups. All calculations were performed using Prism version 3.0.

\section{Results}

\section{OPN Antiserum Neutralizes OPN in vivo}

The glomerular inflammation in our model peaks at approximately 28-35 days, and gradually vanishes. On the other hand, the glomerular fibrosis is histologically detectable as early as days 25-28, and rapidly progresses after day 35. We tested whether blockage of OPN activities at a late inflammatory/early fibrotic stage could still affect inflammation and/or progress of fibrosis. A rabbit OPN antiserum, which effectively inhibited OPN-mediated migration of peritoneal macrophage in in vitro invasion assays, was used to block OPN activity at late inflammatory early fibrotic stage (fig. 1a). Since glomerular fibrosis in our model starts as early as 25 days postimmunization, OPN antiserum was injected intravenously into $8 \mathrm{immu}$ nized rats at days 26, 28, 33 and 38 postimmunization to ensure a constant level of the antibody (fig. 1c). Another group of immunized rats (total 8) received normal rabbit serum (NRS) as controls. Ten immunized rats, without having received any sera, were used as positive controls. Two assays were performed to evaluate effectiveness of the injected OPN antiserum. First, sera OPN concentrations from three groups of rats at day 40 were determined by ELISA. As compared to normal rabbit serum-treated rats, a reduction $(>85 \%)$ in the sera OPN concentration was observed in the OPN antiserum-treated rats, suggesting neutralization of OPN in vivo by the antiserum (fig. 1b). Second, final sera from the OPN antibody-treated rats at day 50 postimmunization showed the presence of rabbit IgG at $26.3 \pm 6.75 \mu \mathrm{g} / \mathrm{ml}$.

\section{OPN Antiserum Treatment Does Not Reduce \\ Glomerular Inflammation}

Flow cytometry analyses were performed on glomerular cells, which were isolated from the OPN- or normal

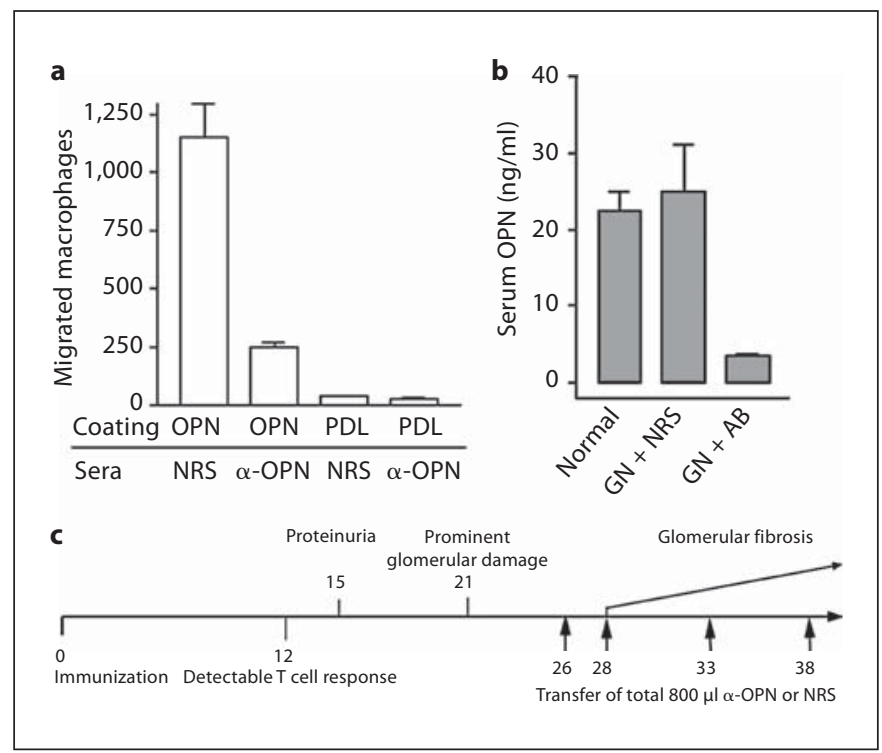

Fig. 1. OPN antiserum neutralizes OPN activities. a OPN-mediated migration of activated peritoneal macrophages after incubation with OPN antiserum ( $\alpha$-OPN) or NRS. Migration is expressed as numbers of macrophages which had migrated through the membrane. PDL = poly D-lysine. $\mathbf{b}$ Sera OPN concentrations in the immunized rats which had received NRS or $\alpha$-OPN. Sera from normal rats were used as a control. c Experimental schedule for OPN antiserum treatment after immunization with nephritogenic T cell epitope pCol(28-40). Immune responses and GN stages are indicated.

serum-treated groups at days 40 and 50. At day 40, 3 rats/ group were analyzed. Both the OPN and normal antiserum groups showed the presence of $\mathrm{CD} 4+\mathrm{T}$ cells and CD11+ cells (mostly macrophages) (fig. 2a-c). Due to high mortality, only 2 rats were available for analysis from the normal sera group at day 50 . In contrast, all rats from the anti-OPN group survived and 3 were used for analysis. At day 50, there were a few, if any, glomerular CD4+ T cells (0.1\%) and CD11+CD8+ cells (0.2\%) in 2 normal rabbit serum controls. On the other hand, the rats from the OPN antiserum group showed the presence of a significant number of CD4+ $\mathrm{T}$ cells $(5.1 \%)$ and CD11+CD8+ cells (1.1\%) (fig. 2a-c). Persistent glomerular inflammation in OPN antiserum-treated rats was further confirmed by immunofluorescence. ED1+ macrophages were observed within the glomeruli of OPN antiserum-treated rats (fig. 2d). However, no macrophages were present within the glomeruli of normal rabbit sera-treated rats except for a few surrounding fibrotic crescents (fig. 2e). Albuminuria was closely monitored daily. There were no significant differences in albu- 
Fig. 2. Treatment of immunized rats with OPN antiserum at late inflammatory/early fibrotic stage does not fully inhibit glomerular inflammation. a, b Flow cytometry on infiltrating leukocytes in the glomeruli of NRS- or $\alpha$-OPN-treated rats at days 40 and 50 postimmunization with pCol(28-40). c Summary of glomerular various cell populations detected by flow cytometry in different groups as indicated. d, e A pair of immunofluorescent images shows the presence of many ED1+ macrophages within a glomerulus of an $\alpha$-OPNtreated rat (c), but not in NRS-treated rats (d). Fibrotic tissue in $\mathbf{d}$ is outlined by arrowheads.

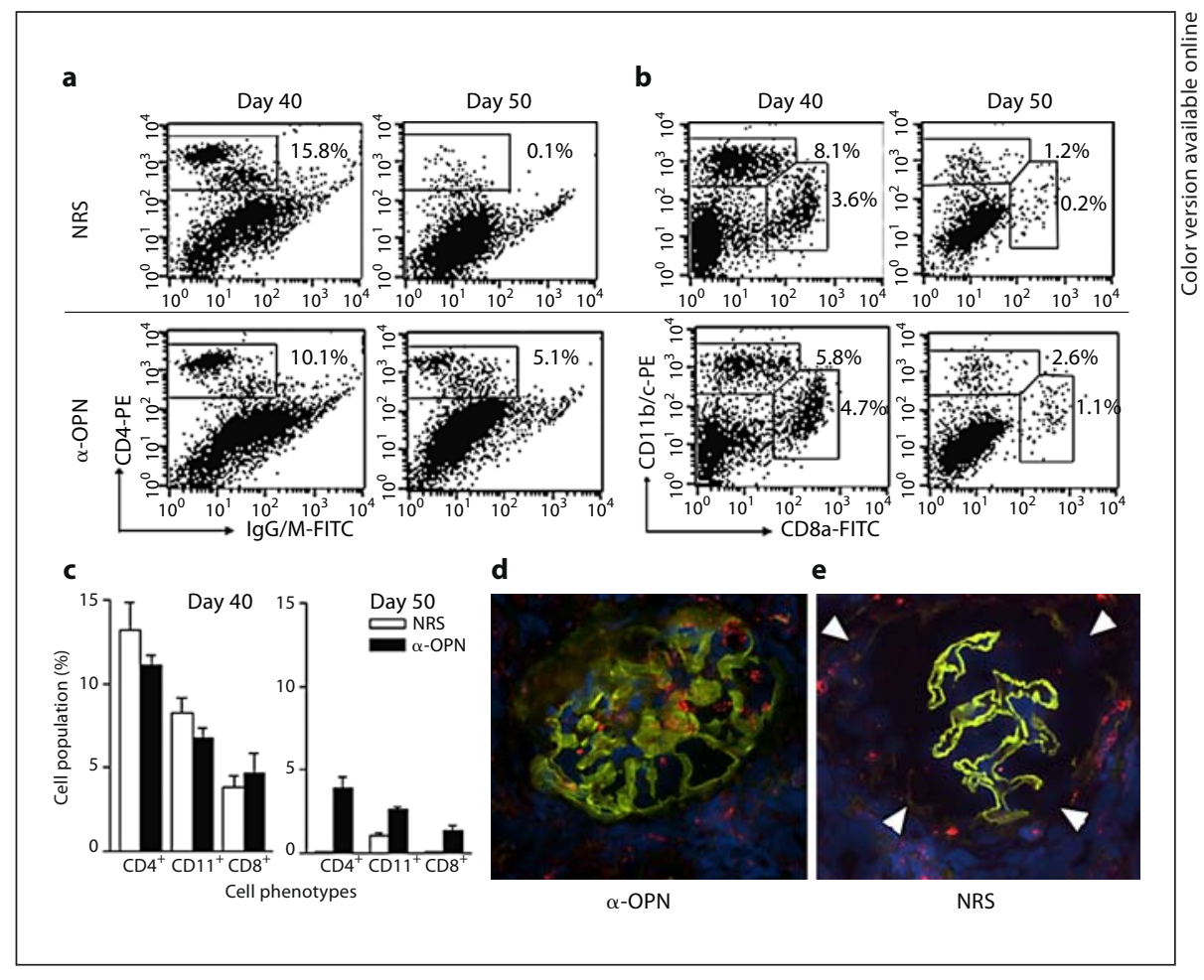

minuria/24-hour or urine albumin concentrations among the two groups during the experiments (fig. 3). However, the majority of normal serum-treated rats developed oligouria after day 40 as indicated by the low urine volumes $(<5 \mathrm{ml})$ per $24 \mathrm{~h}$. On the other hand, oligouria did not occur in OPN antiserum-treated rats. Serum creatinine concentrations at day 50 were determined. Serum creatinine in the OPN antiserum group was elevated ( $2.8 \pm 1.7 \mathrm{mg} / \mathrm{dl}, \mathrm{n}=5)$. However, it was much lower than for the NRS group $(4.6 \pm 0.9 \mathrm{mg} / \mathrm{dl}$, $\mathrm{n}=2$, others died before day 50 ) and the no-treatment group $(4.9 \pm 1.1 \mathrm{mg} / \mathrm{dl}, \mathrm{n}=5$, others died before day 50$)$. The results suggested that OPN antiserum had a limited influence on glomerular inflammation when given at the late inflammatory/early fibrotic stage.

\section{Treatment with OPN Antiserum Reduces Glomerular Fibrosis}

Histopathological analyses, based on specially stained fibrotic tissues, showed that OPN antiserum treatment led to a significant reduction in glomerular fibrosis, both quantitatively and qualitatively (fig. 4a, b). The rats from the OPN antiserum-treated group had a much lower frequency of glomeruli with fibrotic crescents $(39 \%)$ than those from the normal rabbit sera group (79\%) (fig. 4c).

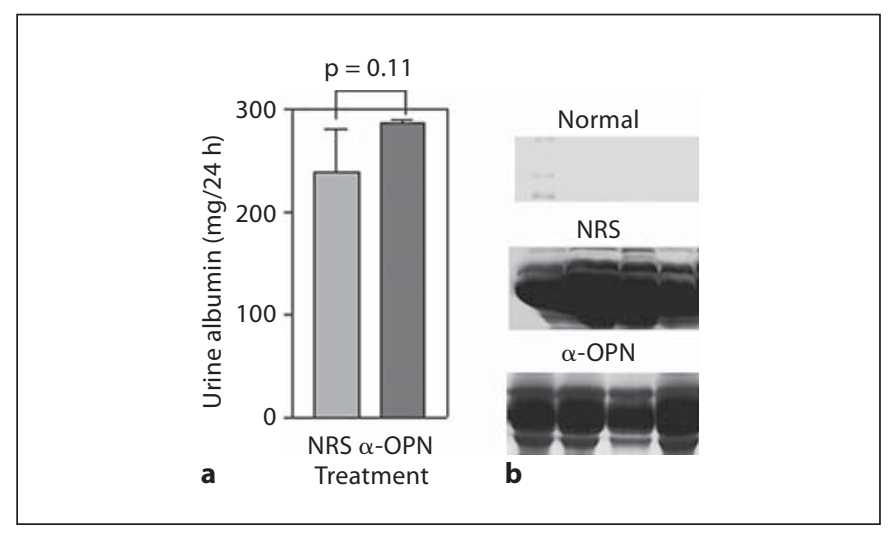

Fig. 3. OPN antiserum treatment does not improve albuminuria in the experimental rats. a Albuminuria, expressed as $\mathrm{mg} / 24 \mathrm{~h}$, NRS- and OPN antiserum-treated rats at day 45 after immunization at their death. b Urine albumin, as detected by SDS-PAGE, in representative rats from different groups.

Although rats from the normal rabbit sera group showed a slightly lower frequency of fibrotic glomeruli than in the group without any sera treatment (86.6\%), there was no significant difference between the two $(p>0.05)$. Furthermore, fibrotic crescents in the OPN antiserum-treat- 

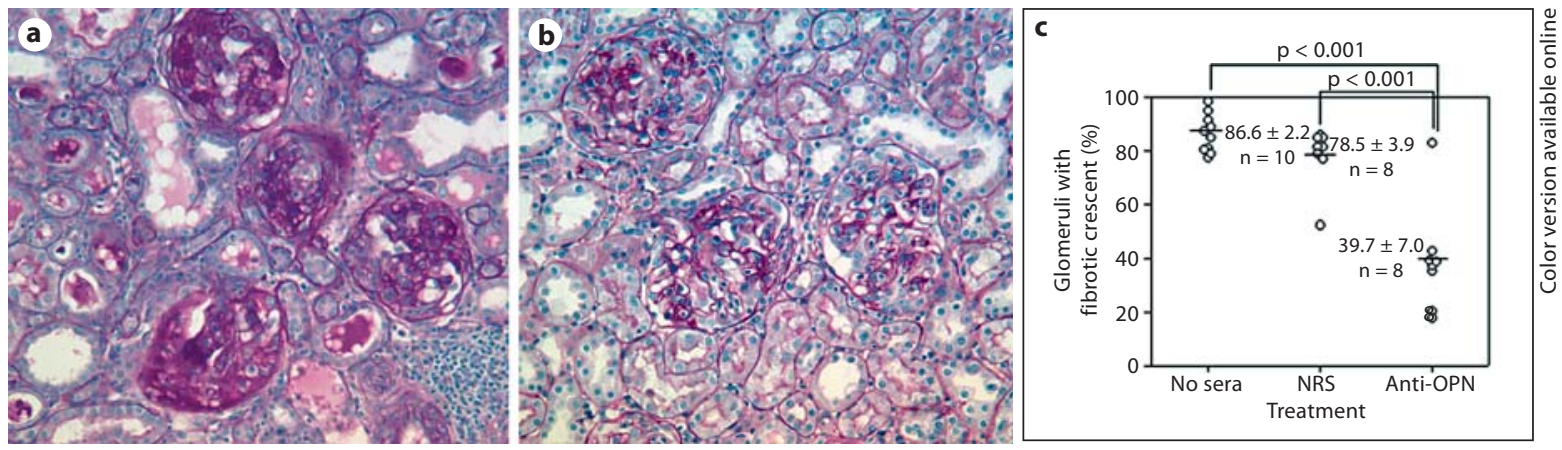

Fig. 4. In vivo treatment with $\mathrm{OPN}$ antiserum reduces severity of fibrosis. $\mathbf{a}$, $\mathbf{b}$ PAS staining at low magnification staining show significantly reduced fibrotic tissues in glomeruli in an OPN antiserum-treated rat (b) as compared to a normal serum control (a). $\times 100$. c Summary of severity of glomerular fibrosis in different experimental groups, expressed as percentage of glomeruli with fibrotic lesions.

ed group were structurally different from the two controls. First, in the two control groups (normal serum or no serum), an entire glomerulus was often occupied by fibrotic tissue without any normal tissue left (fig. 4, 5). Severe glomerular fibrosis greatly affected surrounding tissues, especially the tubules, which had greatly dilated lumens with thin, flattened walls. In contrast, fibrotic tissue was limited to a small area of Bowman's capsule of affected glomeruli in the OPN antiserum-treated animals. Circular crescentic lesions were not observed in any OPN antiserum-treated rats, and no surrounding tissue was affected. Second, in the OPN antiserum-treated rats, glomeruli remained largely intact with most of capillary lumens patent and GBM was not significantly thickened (fig. 5b, d). On the other hand, there was profound destruction of glomeruli in the control groups (fig. 5a, c).

Glomerular fibrosis was next evaluated by collagen $1 \alpha 1$ deposition and expression. Three rats were randomly selected from each group. The renal sections from these rats were stained by anti-collagen $1 \alpha 1$ antibody (fig. 6a, b). Collagen 1 1 1-positive areas in five randomly selected glomeruli from each rat were calculated (fig. 6c). OPN antiserum treatment led to a significantly reduced collagen $1 \alpha 1$-positive area. We next calculated the net increase in the collagen-positive areas using normal glomeruli as a baseline; the positive area in the antiserum-treated rats reduced to $30 \%$ of those from normal serum controls. RTPCR on glomerular RNA showed that all 5 rats treated with OPN antiserum showed a reduced collagen $1 \alpha 1$ expression as compared to those treated with normal rabbit serum, although the reduction level in each OPN antibody-treated individual was variable (fig. 6d). Quantita- tive RT-PCR showed a 6.6 -fold reduction in collagen $1 \alpha 1$ expression in the OPN antiserum-treated group as compared to the normal rabbit sera group.

\section{Discussion}

OPN has been widely recognized as both pro- and anti-inflammatory molecules in various diseases including autoimmune diseases [7-9]. More recently, a critical role of OPN in scar formation or fibrosis in several organs, including kidney, has been reported [10-13]. Previous studies have speculated that OPN played a role in glomerular fibrosis [22]. In the present study, we provided additional evidence that OPN plays an important role in glomerular fibrosis in rat anti-GBM GN. Treatment with an OPN antiserum at the late inflammatory/early fibrosis stage led to a significant reduction in glomerular fibrosis, in both quantity and quality. OPN antibody has been reported to reduce severity of GN in a rat nephrotoxin model when given at an early inflammatory stage [19]. In our study, administration of anti-OPN at the late inflammatory stages did not significantly reduce glomerular inflammation, especially CD4+ cells. Thus, reduction in glomerular fibrosis induced by OPN antiserum in this study may not be mediated by the inhibition of inflammation.

Although several studies, including the present study, suggested a critical role of OPN in glomerular inflammation or renal fibrosis, a previous paper reported that OPN may have no effect on murine anti-GBM GN based on an OPN KO strain [21]. Although it is difficult to address this question, there are a few possibilities which may ex- 

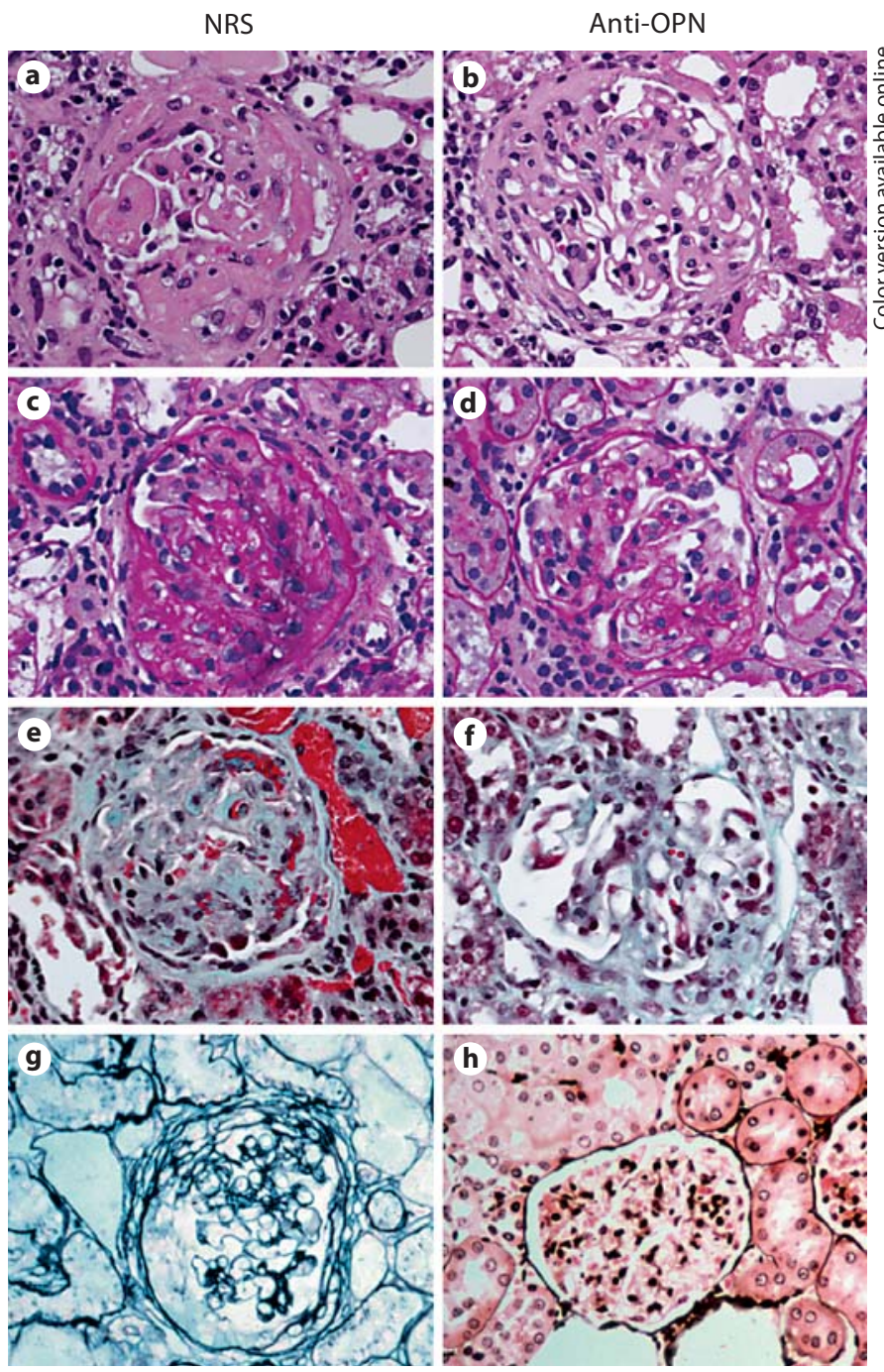

Fig. 5. Histopathology showing fibrotic tissue in glomeruli in OPN antiserum-treated rats (anti-OPN) (b, d, f, h), as compared with NRS-treated controls $(\mathbf{a}, \mathbf{c}, \mathbf{e}, \mathbf{g})$. All rats were previously immunized with pCol(28-40). a, b HE staining; c, d PAS staining; e, $\mathbf{f}$ trichrome staining; $\mathbf{g}, \mathbf{h}$ Jones silver staining. $\times 300$.

plain the conflicting results between the OPN KO model and the antibody blockage model. First, the pathological mechanisms may be different in the two models. In the murine model, glomerulonephritis is induced by an immune response to the 'embedded' foreign antibody bound to GBM [21]. In our model, glomerular inflammation is mediated by the $\mathrm{T}$ cell response to a well-characterized self $\mathrm{T}$ cell epitope from autoantigen collagen $4 \alpha 3$ [24, 25]. Second, the pathological mechanism may differ in different species. Our unpublished data suggest that $\mathrm{T}$ cell re-
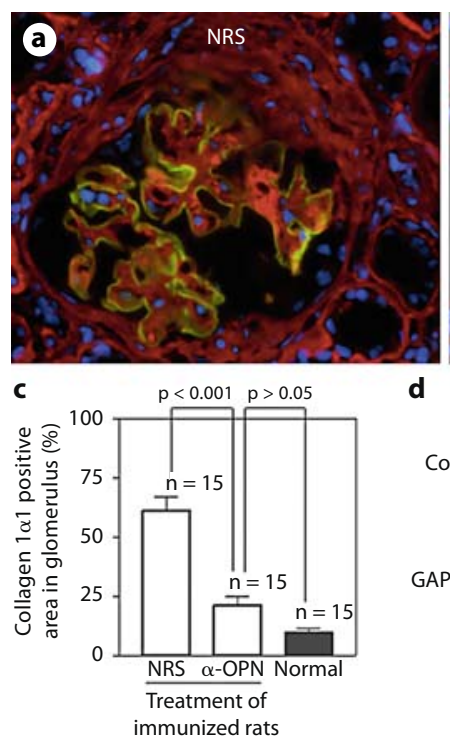

d
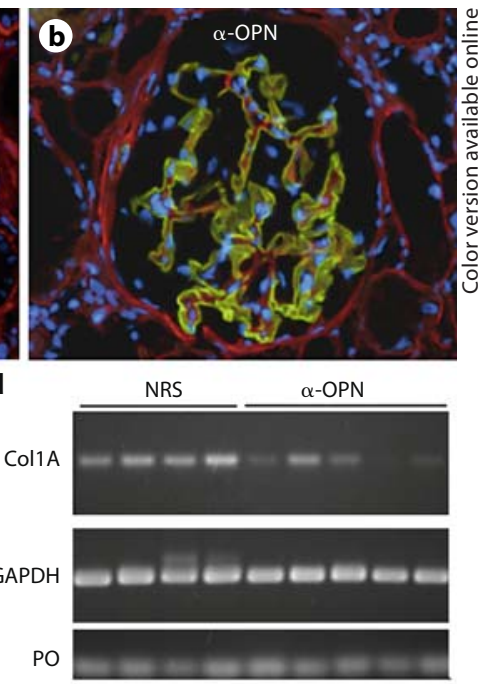

Fig. 6. Reduced collagen $1 \alpha 1$ expression and its deposition in the glomeruli in OPN antiserum-treated rats. a, b Immunofluorescent micrographs show massive deposition of collagen $1 \alpha 1$ (red) in an NRS-treated rat (a), but much less in an $\alpha$-OPN-treated one (b). GBM was counter-stained by SR-13 antibody (green). $\times 500$. c Comparison of collagen $1 \alpha 1$-positive areas in glomeruli in different groups. The kidney sections were stained for collagen $1 \alpha 1$ (see $\mathbf{a}, \mathbf{b})$, and positive areas in a glomerulus were captured using a computer-aided program and calculated as percentage of entire area of a glomerulus. d RT-PCR detection of collagen $1 \alpha 1$ (Col1A) in NRS- or $\alpha$-OPN-treated rats. Two housekeeper genes, GAPDH and acidic ribosomal phosphoprotein (PO), were used for comparison. Each lane represents one individual.

sponse alone may not be sufficient to mediate severe antiGBM GN in mice. Third, it is a well-known phenomenon that in some cases, genetic manipulation may lead to an unexpected result due to complicated regulation/interaction among genes. For example, bone development in OPN KO mice is largely normal.

Different approaches have been tested in experimental models of renal fibrosis. It is clear that TGF- $\beta / \mathrm{Smad}$, as well as angiotensin II and ET1, play a central role in mesangial cell activation and differentiation of fibroblasts to myofibroblasts [4-7]. However, TGF- $\beta$ may no longer be critical once fibrosis is initiated. The fibrotic mechanism downstream of the TGF- $\beta$ pathway has been studied. Several studies suggest a role of ECM, such as SPARC, in renal fibrosis [27]. Several studies have determined OPN's role in renal fibrosis due to ureteral obstruction, ischemia, and cyclosporine toxicity using OPN knockout mice. These studies suggested that OPN may have mul- 
tiple functions including recruitment of macrophages, induction of apoptosis, and modulation of angiotensin II [17]. Our study again demonstrated that OPN could be critical for fibrosis.

However, the mechanism by which OPN antiserum inhibits fibrosis when given at a late inflammatory stage of GN remains unclear in the present study. It is unlikely that inhibition of fibrosis by OPN antiserum is directly related to the TGF- $\beta$ pathway, because OPN antiserum was given after fibrosis had been initiated. One possible mechanism is that reduction in fibrosis is due to inhibition of glomerular inflammation by OPN antibody. However, it is also less likely, as glomerular inflammation was not significantly affected by the antibody. Alternatively, OPN antibody may act at two levels on OPN after fibrosis has been initiated. First, fibrosis, including glomerular fibrosis, is often defined as a wound-healing response that has gone out of control, resulting in excess deposition of ECM. The key cellular mediators are the myofibroblasts, which differentiate from fibroblasts (or glomerular cells such as mesangial cells) and are the main source of excessive ECM proteins $[3,28]$. Interaction with ECM may be required for fibroblasts to differentiate [29]. Thus, blockage of OPN activities in the present study may inhibit differentiation of myofibroblasts. This was evidenced by a much smaller number of fibroblast-like cells in glomeruli in OPN antisera-treated rats [unpubl. data]. Furthermore, our in vitro experiments demonstrated a significantly reduced proliferation rate and altered adhesion in CD90+ glomerular fibroblast-like cells freshly isolated at the early fibrotic stage. Through the analysis of global gene expression, our recent results showed altered expression patterns in fibrosis-related genes [unpubl. data]. In fact, the requirement of OPN for the differentiation of fibroblasts has been demonstrated in the cardiac fibrosis model [14]. Second, OPN may facilitate assembly of ECM protein to form fibrotic tissue. OPN has been recognized as a structural protein with multiple binding sites for collagen and other molecules such as adhesion molecules [30, 31]. A previous study using OPN mutant mice showed a disorganization of matrix in skin incisions, suggesting involvement of OPN in ECM protein assembly [32]. Thus, antibody inhibition of OPN may affect organization of collagen fibers and/or adherence of fibroblasts. However, our antibody blockage experiments were not able to provide direct evidence to support this possibility. We have shown previously a massive deposition of OPN on fibrotic tissue at the mid to late stage of fibrosis [26]. Binding of antibody to OPN may disturb the architecture of fibrotic tissue. This hypothesis can be tested if the OPN antibody is administered after fibrosis has been initiated.

In summary, our study showed that OPN antibody given at the late inflammatory/early fibrotic stage inhibited the progression of fibrosis. The effectiveness of the antibody was not due to inhibition of glomerular inflammation. Several results demonstrated that reduction in fibrosis was through inhibition of glomerular fibroblastlike cell growth/differentiation. OPN has been a therapeutic target in several other fibrotic diseases. Our data showed that the OPN antibody given at late stage of GN was able to halt or inhibit progression of fibrosis after it had been initiated. It remains to be determined, however, whether the antibody treatment may reverse the diseases. Nevertheless, our model will surely be an excellent tool for investigation of the fibrosis mechanism downstream of the TGF- $\beta$ pathway.

\section{Acknowledgements}

This work is supported by the NIH grants R01 DK60029 (Y.H.L.) and R01 DK77857 (Y.-H.L.). We thank Drs. W. Butler, University of Texas Houston and Y. Sado, Okayama University, Japan, for providing antibodies to rat OPN and glomerular basement membrane. Histology support was provided by the Histology Laboratory, Texas Children's Hospital.
References
Chatziantoniou C, Boffa JJ, Tharaux PL, Flamant M, Ronco P, Dussaule JC: Progression and regression in renal vascular and glomerular fibrosis. Int J Exp Pathol 2004;85:1-11.

$\checkmark 2$ Eddy AA: Molecular basis of renal fibrosis. Pediatr Nephrol 2000;15:290-301.

- 3 Strutz F, Zeisberg M: Renal fibroblasts and myofibroblasts in chronic kidney disease. J Am Soc Nephrol 2006;17:2992-2998.

\footnotetext{
4 Border WA, Okuda S, Languino LR, Sporn MB, Ruoslahti E: Suppression of experimental glomerulonephritis by antiserum against transforming growth factor $\beta 1$. Nature 1990; 346:371-374.

5 Gaedeke J, Peters H, Noble NA, Border WA: Angiotensin II, TGF- and Renal Fibrosis; in Wolf G (ed): The Renin-Angiotensin System and Progression of Renal Diseases. Contrib Nephrol. Basel, Karger, 2001, vol 135, pp 153-160.
} 
6 Denhardt DT, Noda M, O’Regan AW, Pavlin D, Berman JS: Osteopontin as a means to cope with environmental insults: regulation of inflammation, tissue remodeling, and cell survival. J Clin Invest 2001;107:1055-1061.

7 Fogo AB: Renal fibrosis: not just PAI-1 in the sky. J Clin Invest 2003;112:326-328.

-8 O’Regan AW, Nau GJ, Chupp GL, Berman JS: Osteopontin (Eta-1) in cell-mediated immunity: teaching an old dog new tricks. Immunol Today 2000;21:475-478.

-9 Bird L: Asthma and allergy: opposing roles for osteopontin. Nature Rev Immunol 2007; $7: 417$.

-10 Chabas D, Baranzini SE, Mitchell M, Bernard CCA, Rittling SR, Denhardt DT, Sobel RA, Lock C, Karpuj M, Pedotti R, Heller R, Oksenberg JR, Steinman L: The influence of the proinflammatory cytokine, osteopontin, on autoimmune demyelinating disease. Science 2001;294:1731-1735.

- 11 Pardo A, Gibson K, Cisneros J, Richards TJ, Yang Y, Becerril C, Yousem S, Herrera I, Ruiz V, Selman M, Kaminski N: Up-regulation and profibrotic role of osteopontin in human idiopathic pulmonary fibrosis. PLoS Med 2005;2:e251.

- 12 Zahradka P: Novel role for osteopontin in cardiac fibrosis. Circ Res 2008;102:270-272.

-13 Mori R, Shaw TJ, Martin P: Molecular mechanisms linking wound inflammation and fibrosis: knockdown of osteopontin leads to rapid repair and reduced scarring. J Exp Med 2008;205:43-51.

- 14 Lenga Y, Koh A, Perera AS, McCulloch CA, Sodek J, Zohar R: Osteopontin expression is required for myofibroblast differentiation. Circ Res 2008;102:319-327.

-15 Xie Y, Sakatsume M, Nishi S, Narita I, Arakawa M, Gejyo F: Expression, roles, receptors, and regulation of osteopontin in the kidney. Kidney Int 2001;60:1645-1657.
16 Mazzali M, Kipari T, Ophascharoensuk V, Wesson JA, Johnson R, Hughes J: Osteopontin - a molecule for all seasons. Q J Med 2002 95:3-13.

17 Wolak T, Kim H, Ren Y, Kim J, Vaziri ND, Nicholas SB: Osteopontin modulates angiotensin II-induced inflammation, oxidative stress, and fibrosis of the kidney. Kidney Int 2009;76:32-43.

18 Lan HY, Yu XQ, Yang N, Nikolic-Paterson DJ, Mu W, Pichler R, Johnson RJ, Atkins RC: De novo glomerular osteopontin expression in rat crescentic glomerulonephritis. Kidney Int 1998;53:136-145.

19 Yu XQ, Fan JM, Nikolic-Paterson DJ, Yang N, Mu W, Pichler R, Johnson RJ, Atkins RC, Lan HY: IL-1 up-regulates osteopontin expression in experimental crescentic glomerulonephritis in the rat. Am J Pathol 1999;154 833-841.

20 Yu XQ, Nikolic-Paterson DJ, Mu W, Giachelli CM, Atkins RC, Johnson RJ, Lan HY: A functional role for osteopontin in experimental crescentic glomerulonephritis in the rat. Proc Assoc Am Phys 1998;110:50-64.

21 Bonvini JM, Schatzmann U, Beck-Schimmer B, Sun LK, Rittling SR, Denhardt DT, Le Hir M, Wüthrich RP: Lack of in vivo function of osteopontin in experimental anti-GBM nephritis. J Am Soc Nephrol 2000;11:16471655.

22 Yoo KH, Thornhill BA, Forbes MS, Coleman CM, Marcinko ES, Liaw L, Chevalier RL: Osteopontin regulates renal apoptosis and interstitial fibrosis in neonatal chronic unilateral ureteral obstruction. Kidney Int 2006; 70:1735-1741.

-23 Wolak T, Kim H, Ren Y, Kim J, Vaziri ND, Nicholas SB: Osteopontin modulates angiotensin II induced inflammation, oxidative stress, and fibrosis of the kidney. Kidney Int 2009;76:32-43.

-24 Wu J, Hicks J, Borillo J, Glass WF 2nd, Lou YH: CD4+ T cells specific to a glomerular basement membrane antigen mediate glomerulonephritis. J Clin Invest 2002;109:517524 .
25 Wu J, Hicks J, Borillo J, Glass WF 2nd, Lou YH: T cell epitope of glomerular basement membrane antigen induces severe glomerulonephritis. Kidney Int 2003;64:1292-1301.

26 Merszei J, Wu J, Torres L, Hicks JM, Bartkowiak T, Tan F, Lou YH: Osteopontin overproduction is associated with progression of glomerular fibrosis in a rat model of antiGBM glomerulonephritis. Am J Nephrol (in press).

27 Alpers CE, Hudkins KL, Segerer S, Sage EH, Pichler R, Couser WG, Johnson RJ, Bassuk JA: Localization of SPARC in developing, mature, and chronically injured human allograft kidneys. Kidney Int 2002;62:20732086.

22 Wynn TA: Cellular and molecular mechanism of fibrosis. J Pathol 2008;214:199-210.

29 Xia H, Nho R, Kleidon J, Kahm J, Henke CA: Polymerized collagen inhibits fibroblasts proliferation via a mechanism involving the formation of a $\beta 1$ integrin-protein phosphatase 2A-tuberous sclerosis complex 2 complex that suppresses S6K1 activity. J Biol Chem 2008;283:20350-20360.

30 Eckes B, Kessler D, Aumailly M, Krieg T: Interactions of fibroblasts with extracellular matrix: implications for the understanding of fibrosis. Springer Semin Immunopathol 2000;21:415-429.

31 Chen Y, Bal BS, Gorsk JP: Calcium and collagen binding properties of osteopontin, bone sialoprotein, and bone acidic glycoprotein-75 from bone. J Biol Chem 1992;267: 24871-24878.

32 Liaw L, Birk DE, Ballas CB, Whitsitt JS, Davidson JM, Hogan BL: Altered wound healing in mice lacking a functional osteopontin gene (spp1). J Clin Invest 1998;101:14681478. 